\title{
A multiband RF signal sampling frequency selection method based on cylindrical surface spectrum analysis
}

\author{
Haijiang Wang ${ }^{1,2^{*}}$, Yangyang Deng ${ }^{1,2}$, Bao Dong ${ }^{1,2}$, Ling Yang ${ }^{1,2}$, Zhendong Yao ${ }^{1,2}$ and Debin Su ${ }^{1,2}$
}

\begin{abstract}
Sampling frequency selection is a key issue in direct digitalization of the radio frequency (RF) signals. In this paper, we propose a cylindrical surface spectrum and arc distance-based sampling frequency selection method for multiband RF signals. In this method, a 3D visual expression of cylindrical surface spectrum of digital signal is introduced. This expression reflects better the circulatory nature of the spectrum of digital signals. It is found more intuitive, heuristic, and effective in dealing with undersampling issues of multiband RF signals than conventional spectrum expressions are. A function of arc distance between two frequency bands are also introduced which turns out to be useful in the determination of sampling frequency and design of digital filters.
\end{abstract}

Keywords: Cylindrical surface spectrum, Arc distance, Multiband, Sampling

\section{Introduction}

In the application of modern electronic technology, sampling from continuous (analog) signal to obtain digital signal has become one of the most common technologies. The relationship between an analog signal and the digital signal sampled from it can be mathematically described by the sampling theorem. As for the baseband signal, the Nyquist criterion requires that the sampling frequency must be more than twice the upper limit of analog signal frequency band, which has long been well known in engineering design. However, with the rapid development of technology, the direct sampling of high frequency signal has become a reality and the Nyquist criterion under literal meaning has long been broken through. What is called "undersampling" technology (which means sampling at a rate below the Nyquist frequency) has been developed. But meanwhile, with the application of software radio idea and the emergence of new types of radar and new communication system, the RF signals and their spectrums are more and more complex. For example, in dual-frequency or multi-frequency

\footnotetext{
* Correspondence: whj@cuit.edu.cn

${ }^{1}$ College of Electronic Engineering, Chengdu University of Information Technology, Chengdu, Sichuan 610225, China

${ }^{2}$ CMA Key Laboratory of Atmospheric Sounding, Chengdu, Sichuan 610225, China
}

radar, the RF echos contain multibands. And in $4 \mathrm{G}$ and the upcoming 5G communications, multicarrier or multiband RF signals are very common. These complex multiband signals pose new challenges to their sampling. In the past two decades, sampling and efficiently reconstructing multiband RF signals has been investigated in many literatures in recent years. The earliest study can be traced to the end of the last century. For example, Raman Venkataramani and Yoram Bresler investigated periodic nonuniform sampling and multichannel processing based sub-Nyquist sampling of multiband signals $[1,2]$; Dennis M. Akos et al. studied direct bandpass sampling of multiple distinct RF signals and proposed a novel technique to determine the absolute minimum sampling frequency for direct digitization of multiple, nonadjacent, frequency bands [3]. Moshe Mishali et al. discussed blind sampling and reconstruction of multiband signals and realized compressive sensing for multiband analog signals with relative small sampling rate under the condition that only the number of bands and their widths are assumed without any other limitations on the frequency support [4], but the theoretical lower bound on the average sampling rate is still twice the minimal rate of knownspectrum recovery. In [5], Satyabrata et.al presented an algorithm to determine the minimum bandpass sampling frequency for direct downconversion of multiple distinct 
RF signals, but in this literature, the iteration-based algorithm was a little complicated and besides, only the case of two frequency bands was considered. In [6], Jie-Cherng Liu reported an efficient method to find the ranges of valid bandpass sampling frequency for direct downconverting multiple bandpass analytic signals (single-sideband RF signals). The algorithm results in the ranges of valid bandpass sampling frequency for the complex signals in terms of bandwidths and band positions of the singlesideband RF signals. But in fact, just as the author said, compared to real bandpass sampling, the valid sampling frequency ranges of the analytic signals are easier to find than those of the real sampling. In recent years, the undersampling of multiband RF signals was still an issue which was frequently researched on, and many meaningful results were achieved [7-10]. For example, in [10], the authors proposed a method to select aliasing-free bandpass sampling frequency in optical phase-modulated and coherent detection (PM-CD) radio-over-fiber (RoF) links, but only two bands were considered. In the existing literatures for multiband signal sampling, all the analysis methods are based on frequency axes under the Cartesian coordinate system. Although the theoretical principle of these methods is still the sampling theorem, because of the disconnectivity between positive and negative frequency bands of the spectrum, getting digital signal spectrum from the analog signal spectrum according to the sampling theorem needs to shift the positive and negative frequency bands many times, which constitutes a complex picture. The key points of the problem are usually concealed by this seeming complexity, making us unable to obtain the essentials easily. Because the sampling problem of this kind of multiband signal has been increasingly common and important, to seek a more simple and clear geometric interpretation will be helpful in sampling and filtering the processing for this kind of signal in concept and even engineering design. So the cylindrical surface spectrum is proposed in this paper.

This tool has the advantage of intuition in concept, similar to the cylindrical phase surface in the oscillation theory and Riemann surface in the theory of complex variable functions [11]. It is more of a tool that is assisting with the thinking and imagination than with the computing. With the assistance of this tool, the main points of the problem sometimes will be revealed more intensively and clearly, so it will even provide some enlightenment to solutions of some problems involving RF signal processing.

With the cylindrical surface spectrum tool, the concept of arc distance between digital signal frequency bands is introduced. This concept has some practical value in quantitative calculation of the sampling frequency selection and filter design for multiband RF signal digitalization.

\section{Derivation of the cylindrical surface spectrum}

Suppose that the continuous-time signal $u(t)$ has the follow relationship with its frequency spectrum $U(f)$

$$
\begin{gathered}
u(f)=\int_{-\infty}^{\infty} u(t) e^{-j 2 \pi f t} d t \\
u(t)=\int_{-\infty}^{\infty} U((f)) e^{j 2 \pi f t} d f
\end{gathered}
$$

Provided that the sampling period is $T_{\mathrm{s}}$, sampling from $u(t)$ can produce the following sequence.

$$
x(n)=u\left(n T_{\mathrm{s}}\right)
$$

The sequence has the following spectrum according to the sampling theorem $[12,13]$.

$$
\begin{aligned}
X(\omega) & =\sum_{n=-\infty}^{\infty} x(n) e^{-j \omega n}=\frac{1}{T_{\mathrm{s}}} \sum_{m=-\infty}^{\infty} U\left(\frac{\omega}{2 \pi T_{\mathrm{s}}}-\frac{m}{T_{\mathrm{s}}}\right) \\
& =f_{\mathrm{s}} \sum_{m=-\infty}^{\infty} U\left(\frac{f_{\mathrm{s}} \omega}{2 \pi}-m f_{\mathrm{s}}\right)
\end{aligned}
$$

In the equation, $f_{\mathrm{s}}=1 / T_{\mathrm{s}}$, representing the sampling frequency.

The familiar result leads us to obtain the spectrogram naturally by making left and right shift of the analog spectrum diagram on the frequency axis infinite time. That is to say, to show digital signal spectrum derived from sampling, more curves should be drawn. It is convenient to handle the sampling problem of the baseband signal in this way because the positive and negative frequency bands of this kind of signal spectrum are mutually connected. Moreover, it is enough to measure the serious degree of aliasing effect by making the right shift of an analog spectrum, and the contribution of further shift terms can be ignored. If we discuss the undersampling problem of the modulating signal, shift terms of the analog spectrum involved will be many. Furthermore, the positive and negative frequency bands of the spectrum for analog signal are mutually separate; consequently, the shifts of the two parts must be taken into account thus making the method of analog spectrum shift no longer an ideal tool for analyzing.

Writing Eq. (3) in an expanded form, we obtain 


$$
\begin{aligned}
X(\omega)= & \cdots+f_{\mathrm{s}} U\left[\frac{f_{\mathrm{s}}}{2 \pi}(\omega+2 \pi)\right] \\
& +f_{\mathrm{s}} U\left(\frac{f_{\mathrm{s}} \omega}{2 \pi}\right)+f_{\mathrm{s}} U\left[\frac{f_{\mathrm{s}}}{2 \pi}(\omega-2 \pi)\right]+\cdots
\end{aligned}
$$

In Eq. (4) we focus on one term equivalent to Eq3. (3) in which $m=0$. For simplicity, the symbol $V(\omega)$ is introduced to express it.

$$
V(\omega)=f_{\mathrm{s}} U\left(\frac{f_{\mathrm{s}} \omega}{2 \pi}\right)
$$

Although $X(\omega)$ is periodic with period of $2 \pi, V(\omega)$ is not, and its shape is consistent with the analog spectrum $U(f)$. On the horizontal axis, however, there is a flexible scale factor, that is

$$
\omega=\frac{2 \pi}{f_{\mathrm{s}}} f
$$

Meanwhile, there is an amplitude factor $f_{\mathrm{s}}$. In this paper, we call $V(\omega)$ the scaled analog spectrum in the later parts. When using the symbols in Eq. (5), Eq. (4) can be expressed as

$$
\begin{aligned}
X(\omega) & =\cdots+V(\omega+2 \pi)+V(\omega)+V(\omega-2 \pi)+\cdots \\
& =\sum_{k=-\infty}^{\infty} V(\omega+2 k \pi)
\end{aligned}
$$

Firstly, only two terms in Eq. (7) are taken into consideration that is $V(\omega)$ and $V(\omega+2 \pi)$. In Fig. 1a, as for a length of amplitude magnitude of one signal's scaled analog spectrum in the interval $(-\pi, \pi],|V(\omega)|$ is drawn by a solid line. At the same time, $|V(\omega+2 \pi)|$ is drawn by a dotted line. Suppose that $\omega_{1}$ is any point in the interval $(-\pi, \pi]$, and its corresponding amplitude magnitude $\left|V\left(\omega_{1}\right)\right|$ is expressed by $P$, while $\left|V\left(\omega_{1}\right)+2 \pi\right|$ is expressed by $P_{1}$. Their corresponding complex number $V(\omega)$ and $V(\omega+2 \pi)$ should be added together according to Eq. (7). Otherwise, the value $|V|$ whose abscissa is $\omega_{1}+2 \pi$ in the interval $(\pi$, $3 \pi$ ] also equals to $\left|V\left(\omega_{1}+2 \pi\right)\right|$, and it can be expressed by $Q_{1}$. Suppose that we cut down this strip of paper on which the scaled analog spectrum in the interval $(-\pi, \pi]$ is drawn and stick it on a cylinder whose cross section is a unit circle in the following way. Fix the origin of coordinates $(0,0)$ on a point of $R$ on the cylinder, and make the vertical coordinate direction parallel to the axis of the cylinder. Furthermore, place the horizontal coordinate $\omega$ along the unit circle of the cylindrical cross section, which is perpendicular to the ordinate axis. The value of the horizontal coordinate $\omega$ is equal to the arc length from the origin point to this abscissa point. Consequently, it is equal to the angle between the vector $\overline{O R}$ and the vector from the center of a unit circle $\mathrm{O}$ to this abscissa point. For the purpose of clarity, the abscissa that is fixed on the cylindrical surface is called circumferential coordinate, and the ordinate is called axial coordinate. Obviously, this paper just fully covers the cylinder surface because the width of the paper along the horizontal axis is exactly $2 \pi$. Next, choose the strip of paper on which the scaled analog spectrum in the interval $(-\pi, 3 \pi]$ is drawn, and fix it on the former paper in the same way to make them coincide with each other. Suppose that the pieces of paper are transplant, then the situation shown in Fig. 1b can appear, and $P_{1}$ overlaps together with $Q_{1}$ by now.

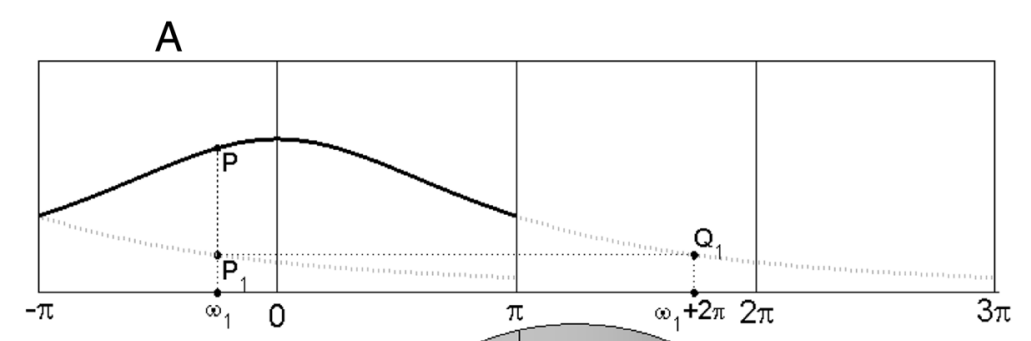

B

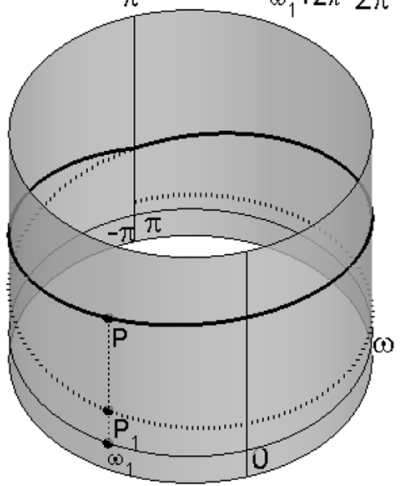

Fig. 1 The derivation of the cylindrical surface spectrum: (a) digital signal spectrum obtained by shifting the analog spectrum and (b) the cylindrical surface expression of the digital signal spectrum 
Thinking about the other terms in Eq. (7), we repeat the above steps. The transparent piece of paper in the interval $((2 k-1) \pi,(2 k+1) \pi]$ displays the amplitude magnitude of $V(\omega+2 k \pi)$, which is any term in Eq. (7). As the value of $k$ increases, the whole scaled analog spectrum $|V(\omega)|$ can be continuously expressed on the cylindrical surface. Therefore, the way of using a single curve and twining the cylindrical surface many layers replaces the way of using each shift term to represent the digital signal spectrum. By now, the aliasing effect is shown by twining the cylindrical surface with the same curve: more than one axis coordinate which are not equal to zero appear on the same circle coordinate of the curve. As a result of the addition of their corresponding complex number value, the shape of the digital spectrum is no longer similar with the spectrum of the analog signal used for sampling.

As a three-dimensional space curve, the expression on the cylindrical surface for the amplitude magnitude of the scaled analog spectrum is a three-dimensional vector type, and $\omega$ is used as its parameter.

$$
r(\omega)=\left[\begin{array}{ll}
\cos \omega, & \sin \omega,|V(\omega)|]
\end{array}\right]
$$

As we see from Eqs. (5) and (6), the scale analog spectrum $V(w)$ is proportional to the analog spectrum $U(f)$. The spectrum on the cylindrical surface of the sampled digital signal can be derived from the spectrum of the analog signal directly. If we are only interested in the position and shape of the spectrum, then

$$
r(f)=\left[\cos \frac{2 \pi f}{f_{\mathrm{s}}}, \quad \sin \frac{2 \pi f}{f_{\mathrm{s}}},|U(f)|\right]
$$

The following example can demonstrate it. Suppose that an analog signal has the spectrum $U(f)$, as depicted in Fig. 2a. Obviously, it contains a baseband signal whose bandwidth of double sideband is $B_{0}=10 \mathrm{KHz}$ and two modulating signals. Among the two modulating signals, the carrier frequency and modulation bandwidth of one is $f_{1}=20 \mathrm{KHz}, B_{1}=5 \mathrm{KHz}$, and of the other is $f_{2}=40 \mathrm{KHz}, B_{2}=8 \mathrm{KHz}$. If the sampling frequency is $30 \mathrm{KHz}$, its spectrum on the cylindrical surface can be drawn according to Eq. (9), as illustrated in Fig. 2 b.

It can be seen from the figure above, at such sampling rate, although in digital frequency domain, the two modulating signals are separate from the baseband signal, they overlaps and cannot be separated by filtering. We will return back to use this example in the fourth section, and use the arc distance of frequency band to obtain the appropriate sampling rate, so as to separate the signals.

The meaning of Nyquist criterion is expressed by the cylindrical surface spectrum in Fig. 3. What Fig. 3a expresses is a baseband signal whose highest frequency is below half the sampling frequency. Its cylindrical surface spectrum is depicted in Fig. 3b. Because digital signal spectrum cannot cover the whole cylindrical surface, there will not be aliasing at this time. The same analog signal is illustrated in Fig. 3c; however, the half sampling frequency is below the highest frequency of the spectrum. Then the digital spectrum enters into another cylindrical layer from its original "layer", consequently, the aliasing occurs, as illustrated in phase 3D.

\section{The arc distance between the digital signal spectrum}

As we can see from introducing the cylindrical surface spectrum, after multiplying the horizontal axis $f$ by a scale factor $2 \pi T_{\mathrm{s}}\left(T_{\mathrm{s}}=1 / f_{\mathrm{s}}\right.$ is the sampling period), the scaled coordinate axis obtained is twined around the unit circle as

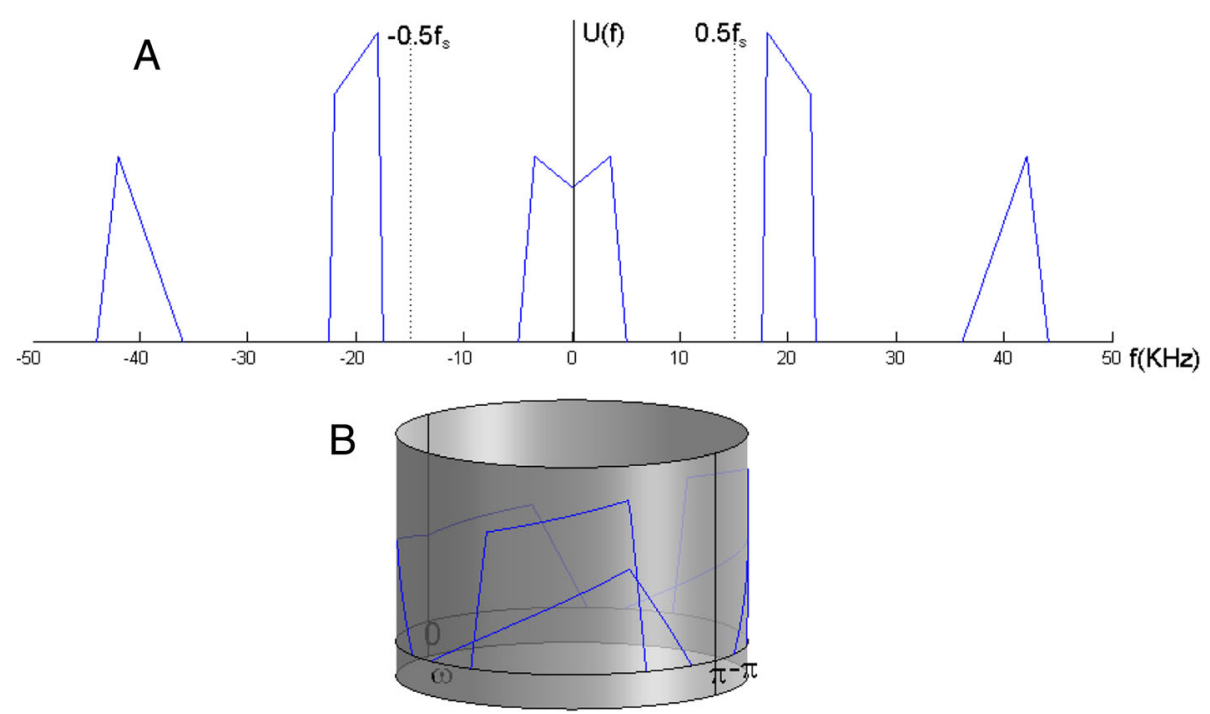

Fig. 2 a The analog spectrum of a signal before sampling and $\mathbf{b}$ the cylindrical surface spectrum of the signal after sampling 


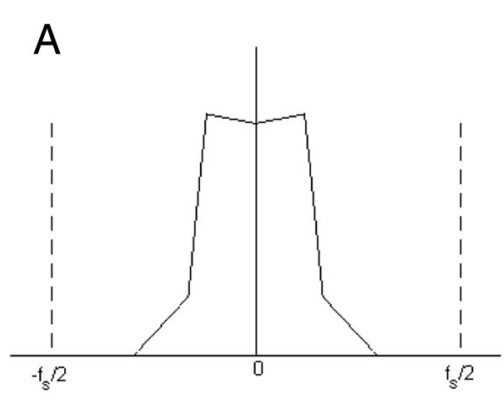

B
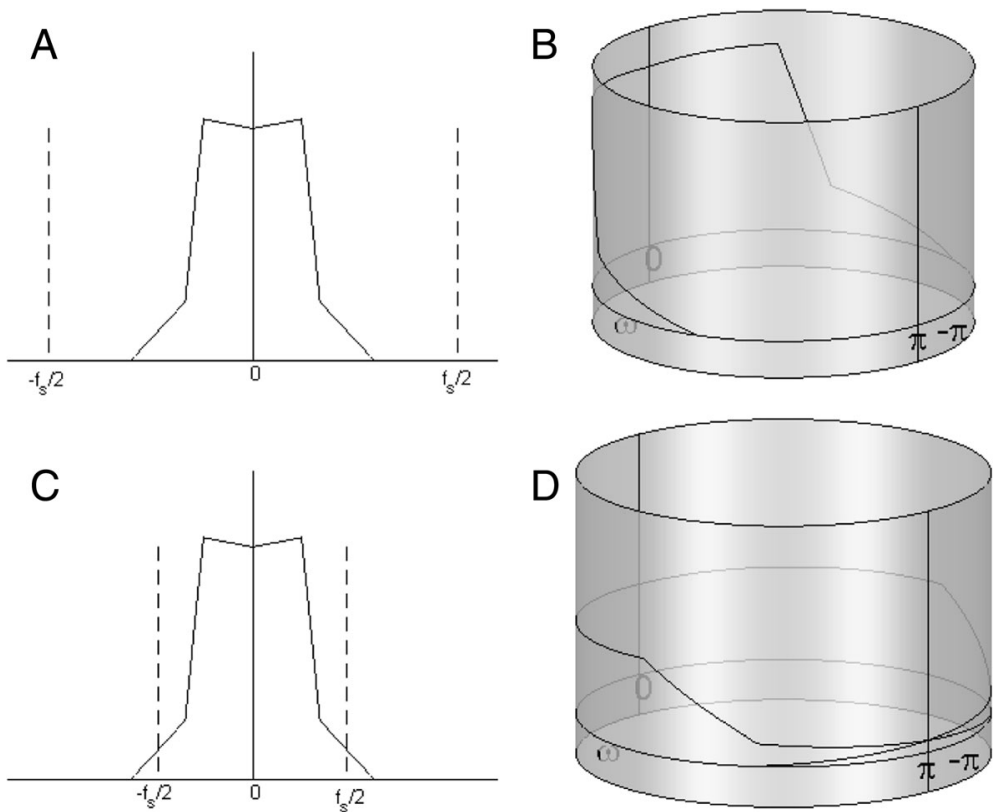

Fig. 3 The Nyquist criterion described in cylindrical surface spectrum: (a) the analog spectrum of a baseband signal whose highest frequency is lower than half of the sampling frequency; (b) the corresponding cylindrical surface spectrum of (a) after sampling; (c) the analog spectrum of a baseband signal whose highest frequency is higher than half of the sampling frequency; (d) the corresponding cylindrical surface spectrum of (c) after sampling

the cylindrical cross section, and we can also see it from Eq. (6). Suppose that the analog frequency $f_{2}>f_{1}$, then their corresponding points on the unit circle are $z_{1}$ $=e^{j 2 \pi I_{s} f_{1}}$ and $z_{2}=e^{j 2 \pi T_{s} f_{2}}$, respectively. Moreover, the arc distance between the two points is defined as the absolute value of the argument of the quotient of the two complex numbers.

$$
\begin{aligned}
D_{\mathrm{c}} & =|\arg (z 2 / z 1)| \\
& =\pi\left|\bmod \left(2 T_{\mathrm{s}}\left(f_{2}-f_{1}\right)+1,2\right)-1\right|
\end{aligned}
$$

To simplify the calculation, the arc length of the semicircle is served as the calculation unit, then the factor $\pi$ at the right most of the above equation can be eliminated. Moreover, among the filter calculating functions in MATLAB, it is used as the unit of the digital frequency.
The arc distance between the two digital frequencies changes when their corresponding analog frequency difference changes, and the changing law is illustrated in Fig. 4.

Now, we discuss the arc distance between the two digital frequency bands. The arc distance between the two frequency points has been defined in Eq. (10), and the frequency band can be regarded as a set of points inside the distance space. According to the principle of set theory, suppose that there is any point $u_{1} \in U_{1}$ and any point $u_{2} \in U_{2}$, the distance between the two sets of points $U_{1}$ and $U_{2}$ is the greatest lower bounding of the distance $d\left(u_{1}, u_{2}\right)$ : $d\left(U_{1}, U_{2}\right)=\inf \left\{d\left(u_{1}, u_{2}\right): u_{1} \in U_{1}, u_{2} \in U_{2}\right\}$. Suppose that there are two analog signals, and their center frequency are $f_{01}$ and $f_{02}$, and their bandwidth are $B_{1}$ and $B_{2}$,respectively. The corresponding arc distance of the two center frequency is

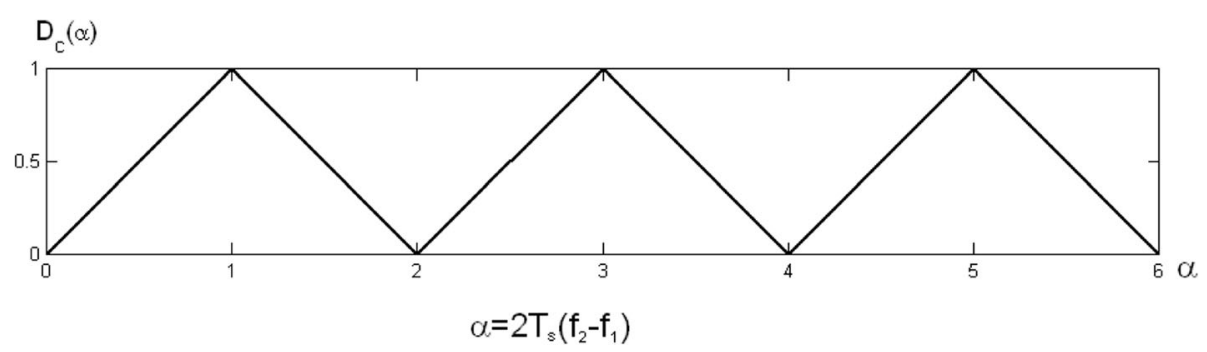

Fig. 4 The relationship between the arc distance of the digital frequencies and the corresponding analog frequency difference of two signals 


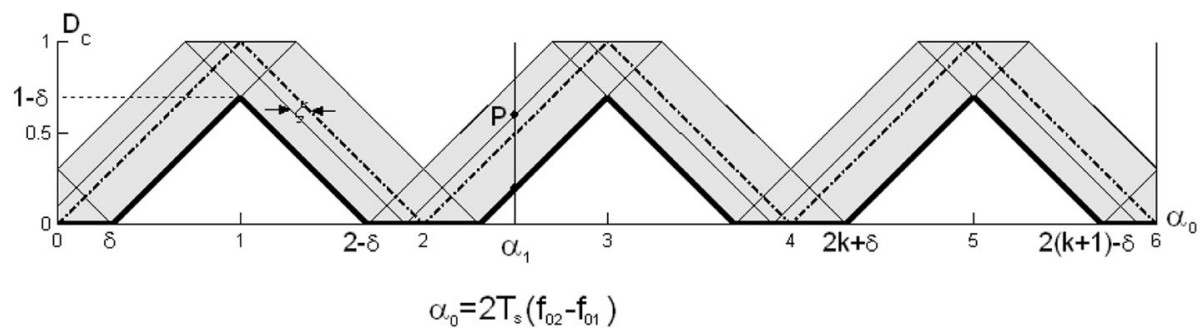

Fig. 5 The arc distance between two digital frequency bands

$$
\begin{aligned}
D_{\mathrm{c} 0} & =\left|\bmod \left[2 T_{\mathrm{s}}\left(f_{02}-f_{01}\right)+1,2\right]-1\right| \\
& =\left|\bmod \left(\alpha_{0}+1,2\right)-1\right|
\end{aligned}
$$

$D_{\mathrm{co} 0}\left(\alpha_{0}\right)$ has been depicted by dotted dash lines in Fig. 5 . Now, we take into account the arc distance between the corresponding digital frequency of any point $f_{01}+\Delta f_{1}$ and $f_{02}+\Delta f_{2}$. They are in the interior of frequency band 1 and frequency band 2 .

$$
\begin{aligned}
D_{\mathrm{c}} & =\left|\bmod \left[2 T_{\mathrm{s}}\left(f_{02}+\Delta f_{2}-f_{01}-\Delta f_{1}\right)+1,2\right]-1\right| \\
& =\left|\bmod \left[2 T_{\mathrm{s}}\left(f_{02}-f_{01}\right)+2 T_{\mathrm{s}}\left(\Delta f_{2}-\Delta f_{1}\right)+1,2\right]-1\right| \\
& =D_{\mathrm{c} 0}\left(\alpha_{0}+\xi\right)
\end{aligned}
$$

In the equation, $\xi=2 T_{\mathrm{s}}\left(\Delta f_{2}-\Delta f_{1}\right)$. Let $\delta=T_{\mathrm{s}}\left(B_{1}+B_{2}\right)$, obviously, $-\delta \leq \xi \leq \delta$.

That is to say, by shifting the arc distance function $D_{\text {co }}\left(\alpha_{0}\right)$ of the center frequency a amount of $\xi$, the arc distance between any two points inside the frequency band can be obtained. Under the condition of $-\delta \leq \xi \leq \delta$, change the value to obtain a curve family which moves horizontally, as illustrated in the shaded area in Fig. 5. For the given abscissa $\alpha_{0}=\alpha_{1}$, the distance between the frequency band is the minimum value between its corresponding vertical coordinates (the point $P$ in Fig. 5) among the curve family. Change the abscissa $\alpha_{1}$ and to obtain the function as expressed by a heavy line in Fig. 5, and the heavy line is just the lower boundary of the shaded area. The process is as follows: move $D_{\mathrm{c} 0}\left(\alpha_{0}\right)$ down an amount of $\delta$ and replace the negative value by zero.

$$
\begin{aligned}
D_{\mathrm{c} B}\left(\alpha_{0}, \delta\right) & =\max \left(0, \quad \bmod \left(\alpha_{0}+1,2\right)-1 \mid-\delta\right) \\
& =\max \left(0, \mid \bmod \left[2 T_{\mathrm{s}}\left(f_{02}-f_{01}\right)\right.\right. \\
& \left.+1,2]-1 \mid-T_{\mathrm{s}}\left(B_{1}+B_{2}\right)\right)
\end{aligned}
$$

\section{Simulation and discussion of undersampling of the narrow-band signal}

As one application example, we will discuss the undersampling problem of the narrow-band signal. Suppose that the carrier frequency of the narrow-band signal is $f_{\mathrm{c}}$, the bandwidth is $\mathrm{B}$, and the sampling period is $T_{\mathrm{s}}$. Then, $\alpha_{0}=2 T_{\mathrm{s}}\left[f_{\mathrm{c}}-\left(-f_{\mathrm{c}}\right)\right]=4 T_{\mathrm{s}} f_{\mathrm{c}}, \delta=2 T_{\mathrm{s}} B$. The arc distance between its two frequency bands which are mirror symmetric is

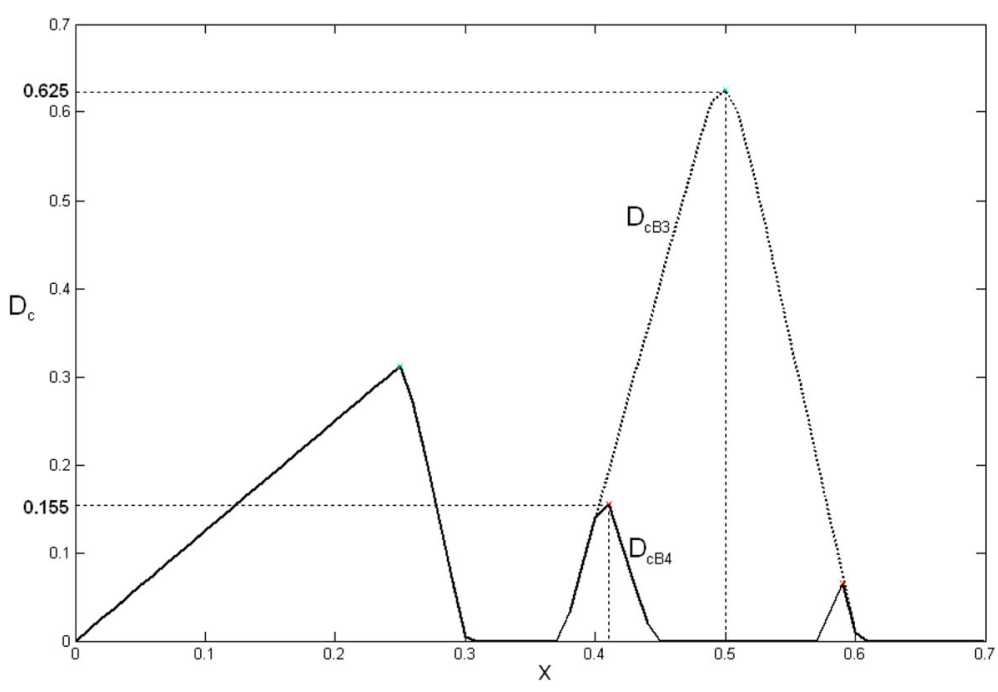

Fig. 6 Searching of the arc distance peaks with the variation of $f_{1} T_{s}$ 


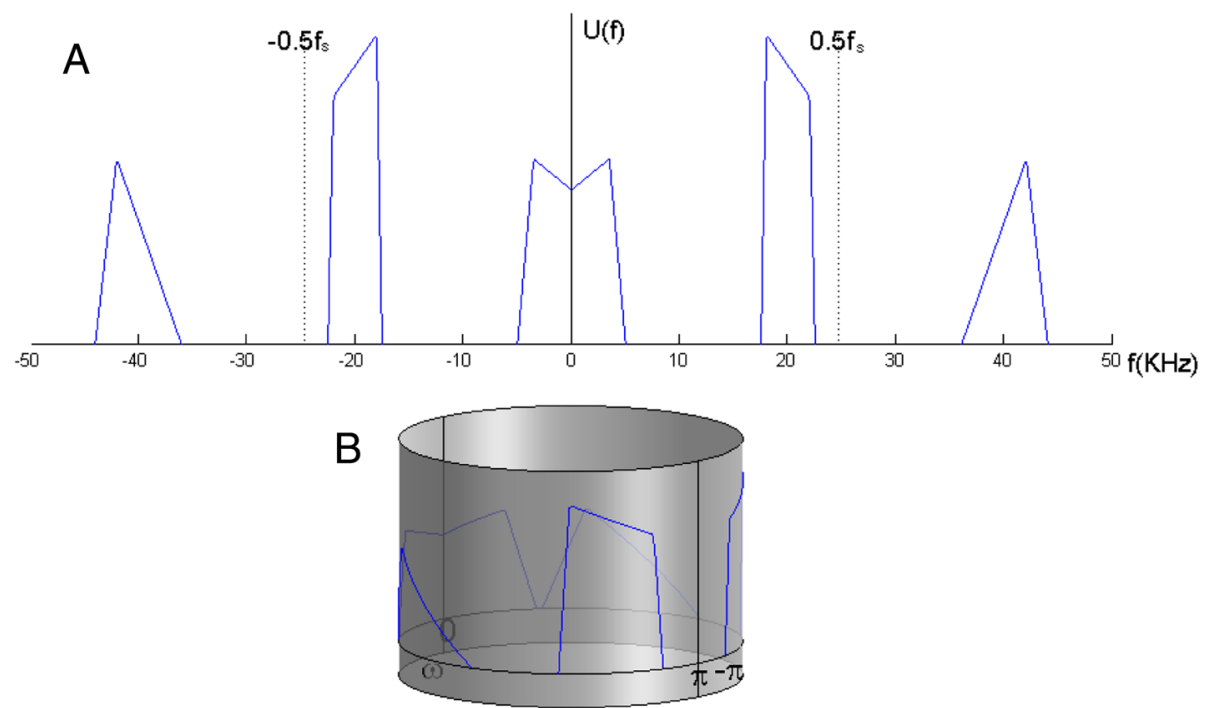

Fig. 7 The cylindrical surface spectrum (b) of a multiband signal (a) when the minimum arc distance is 0.155

$$
\begin{aligned}
D_{\mathrm{c} B}\left(4 T_{\mathrm{s}} f_{\mathrm{c}}, 2 T_{\mathrm{s}} B\right)= & \max \left(0, \quad \mid \bmod \left(4 T_{\mathrm{s}} f_{\mathrm{c}}\right.\right. \\
& \left.+1,2)-1 \mid-2 T_{\mathrm{s}} B\right)
\end{aligned}
$$

Because the maximum value of the function $D_{\mathrm{c} B}\left(\alpha_{0}, \delta\right)$ is $1-\delta$, it must be positive-valued. Otherwise, $D_{\mathrm{c} B}\left(\alpha_{0}, \delta\right) \equiv 0$, meaning that the two mirror frequency bands are always overlapping with each other, and it is impossible to separate them. This request can be expressed as $\delta=2 T_{\mathrm{s}} B<1 \Rightarrow f_{\mathrm{s}}>2 B$.

Because $D_{\mathrm{C} B}\left(\alpha_{0}, \delta\right)$ is a periodic function of $\alpha_{0}$, its area which is greater than zero appears periodically on the $\alpha_{0}$ axis. Such kth area is( illustrated in Fig. 5)

$$
2 k+\delta<\alpha_{0}<2(k+1)-\delta
$$

The already known results can be derived from it.

$$
\frac{k}{2} f_{s}+\frac{B}{2}<f_{c}<\frac{k+1}{2} f_{s}-\frac{B}{2}
$$

Let us go back to the example mentioned in the second section. As can be seen in Eq. (13), the arc distance between the frequency band is a function of five variables $D_{\mathrm{c} B}\left(f_{01}, B_{1}, f_{02}, B_{2}, T_{s}\right)$. If the positive frequency bands of the modulated signal whose center is $f_{1}$ needs to be extracted, we can calculate the distance between this
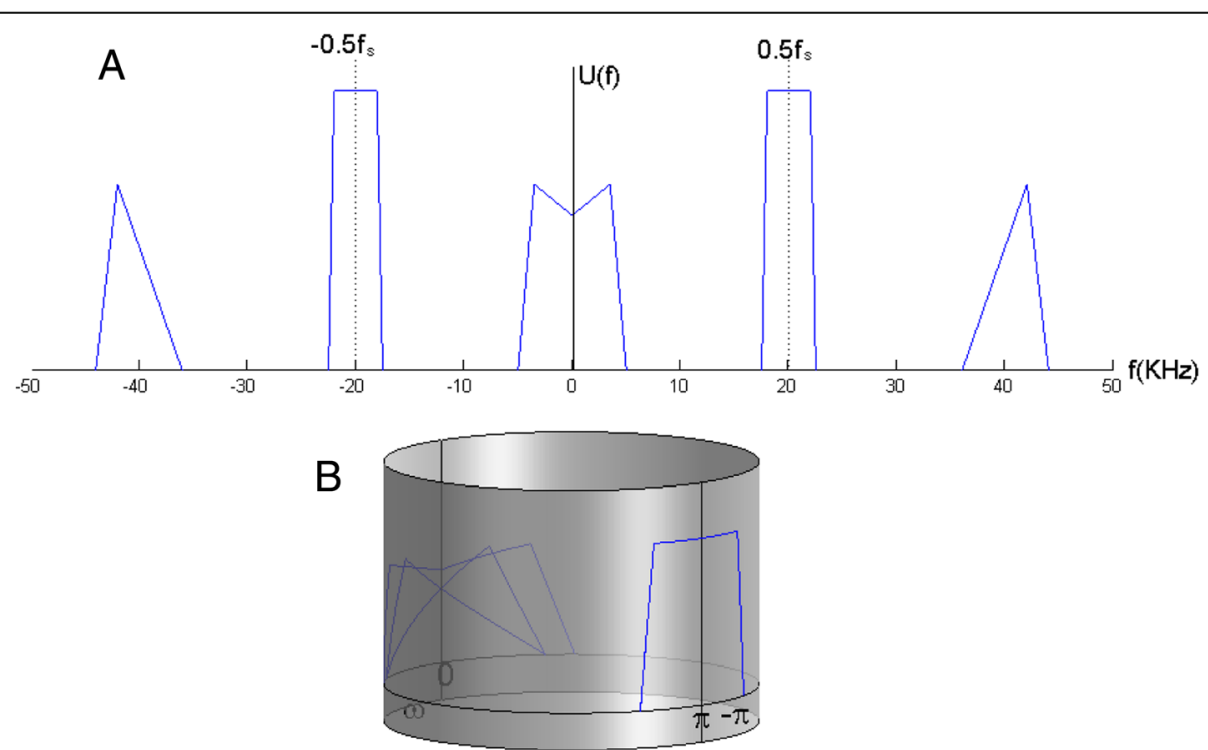

Fig. 8 The cylindrical surface spectrum (b) of a multiband signal (a) when the minimum arc distance is 0.625 
frequency band and other four frequency bands. The parameter $x=f_{1} T_{s}$ is introduced to reflect the change of the sampling period.

$$
\begin{aligned}
D_{\mathrm{c} 1} & =D_{\mathrm{c} B}\left(f_{1}, B_{1}, \quad f_{2}, \quad B_{2}, x / f_{1}\right) ; D_{\mathrm{c} 2} \\
& =D_{\mathrm{c} B}\left(f_{1}, B_{1}, \quad 0, \quad B_{0}, x / f_{1}\right) ; \\
D_{\mathrm{c} 3} & =D_{\mathrm{c} B}\left(f_{1}, B_{1}, \quad-f_{2}, \quad B_{2}, x / f_{1}\right) ; D_{\mathrm{c} 4} \\
& =D_{\mathrm{c} B}\left(f_{1}, B_{1}, \quad-f_{1}, B_{1}, x / f_{1}\right) ;
\end{aligned}
$$

In order to separate the frequency band $f_{1}$ and other four frequency bands, the minimum arc distance value should be calculated.

$$
D_{\mathrm{c} B 4}(x)=\min \left(D_{\mathrm{c} 1}, D_{\mathrm{c} 2}, D_{\mathrm{c} 3}, D_{\mathrm{c} 4}\right)
$$

Then, to find out the maximum value of $D_{\mathrm{c}}(x)$, the parameter $x$ should be varied.

The above-mentioned function is illustrated by the solid line in Fig. 6. Its first extreme point appears when $x=0.25$, and its corresponding sampling frequency is $80 \mathrm{KHz}$. This is close to the Nyquist frequency under the literal meaning. Obviously, it is not the ideal choice. The second extreme point appears when $x=0.41$, and its corresponding sampling frequency is $48.7805 \mathrm{KHz}$. The shortest arc distance between the frequency band expected to extract and the other frequency bands is 0.155 . At this moment, the cylindrical surface spectrum is illustrated in Fig. 7. As can be seen from the figure, the frequency band $f_{1}$ that is expected to extract has been separated from the other frequency bands. If this frequency band needs to be extracted, the quadrature demodulation must be conducted, and then, it should pass the low-pass filter. The shortest arc distance value 0.155 is the main basis which determines the transition bandwidth of the filter.

The assumption above is that the signal spectrum does not have the symmetry about the center frequency, meaning that it contains the information of phase. In order to obtain this information, the complex-valued signal needs to be obtained firstly. However, if the frequency band $f_{1}$ is a simply modulated audio signal, then the spectrum has the symmetry about the center frequency, that is to say, the two mirror frequency bands can overlap together. Thus, after removing the the arc distance between the signal band and its mirror frequency bands, the arc distance in Eq. (17) turns out to be

$$
D_{\mathrm{c} B 3}(x)=\min \left(D_{\mathrm{c} 1}, D_{\mathrm{c} 2}, D_{\mathrm{c} 3}\right)
$$

Its value with the change of $x$ is illustrated by the dotted line in Fig. 6. Its extremum value is 0.625 , and its corresponding sampling frequency is $40 \mathrm{KHz}$. The cylindrical surface spectrum of the signal is illustrated in Fig. 8. At this moment, the demodulation will be very simple. In order to rotate the frequency spectrum an angle of $180^{\circ}$, the sequence only needs to be multiplied by 1 and -1 alternately. Then the low-pass filter should be used to deal with the signal. Because the shortest arc distance of the frequency band needed to be filtered is as big as 0.625 , the design of the filter will be very easy.

\section{Conclusions}

As can be seen from the discussion above, the cylindrical surface spectrum reflects the periodicity of the digital spectrum more naturally and intuitively. Consequently, it becomes an effective and powerful tool in analyzing and designing the sampling and processing problems of the high-frequency signals. Obviously, they can help us form a clear and intuitive point of view about such problems.

The cylindrical surface spectrum can better reflect the existing operation of the digital signal processing such as demodulation and filtering more simply. Moreover, it can reflect the inner connection of all kinds of filtering characteristics more effectively.

With the help of MATLAB, the translucent cylindrical plotting becomes easy and workable, so it has the potential for popularizing. In addition, the concept of the arc distance between the signal bands is very useful in the sampling rate determination and the filter design.

\section{Funding}

Research for this paper was funded by the Science and Technology Department of Sichuan Province (Project 2016JY0106) and the Education Department of Sichuan Province (Project 16ZA0209) of China.

\section{Competing interests}

The authors declare that they have no competing interests.

Received: 12 July 2016 Accepted: 28 November 2016

Published online: 07 December 2016

\section{References}

1. R Venkataramani, Y Bresler, Sub-Nyquist sampling of multiband signals: perfect reconstruction and bounds on aliasing error. Proceedings of IEEE Int. Conf. on Acoustics, Speech, and Signal Processing, ICASSP '98 3, 1633-1636 (1998)

2. R Venkataramani, Y Bresler, Perfect reconstruction formulas and bounds on aliasing error in sub-Nyquist nonuniform sampling of multiband signals. IEEE Trans. on Inform Theory 46(6), 2173-2183 (2000)

3. DM Akos, M Stockmaster, JBY Tsui, J Caschera, Direct bandpass sampling of multiple distinct RF signals. IEEE Trans. on Communications 47(7), 983-988 (1999)

4. M Mishali, YC Eldar, Blind multiband signal reconstruction: compressed sensing for analog signals. IEEE Trans. on Signal Processing 57(3), 993-1009 (2009)

5. S Sen, VM Gadre, An Algorithm for Minimum Bandpass Sampling Frequency for Multiple RF Signals in SDR System. IEEE/SP 13th Workshop on Statistical Signal Processing, 2005, pp. 327-332

6. JC Liu, Complex bandpass sampling and direct down conversion of multiband analytic signals. Signal Processing 90(2), 504-512 (2010)

7. Q Wei, T Jin, F Yu, Effective frequency selection algorithm for bandpass sampling of multiband RF signals based on relative frequency interval. 2010 International Conference on Computer Application and System Modeling, ICCASM'2010 10, 431-435 (2010)

8. JM Muñoz-Ferreras, R Gómez-García, F Pérez-Martínez, RF front-end concept and implementation for direct sampling of multiband signals. IEEE Trans. on Circuits and Systems-II: Express Briefs 58(3), 129-133 (2011)

9. Y Chen, AJ Goldsmith, YC Eldar, Minimax Universal Sampling for Compound Multiband Channels. 2013 IEEE International Symposium on Information Theory, ISIT'2013, 2013, pp. 1032-1036 
10. M. Cao, J. Li, F. Yin, Y. Dai, K. Xu, Digital coherent detection with bandpass sampling in multiband phase-modulated radio-over-fiber Links. 2014 13th International Conference on Optical Communications and Networks, ICOCN'2014, pp. 1-4, 9-10 (2014).

11. SN Elaydi, Oscillation Theory (Springer, New York, 1999)

12. AV Oppenheim, RW Schafer, Discrete-time signal processing (Prentice-Hall, Englewood, Cliffs, 1989)

13. SK Mitra, Digital Signal Processing, A computer-based approach (McGraw-Hill\& Tsinghua University Press, Beiijng, 2001)

\section{Submit your manuscript to a SpringerOpen ${ }^{\circ}$ journal and benefit from:}

- Convenient online submission

- Rigorous peer review

- Immediate publication on acceptance

- Open access: articles freely available online

- High visibility within the field

Retaining the copyright to your article

Submit your next manuscript at $>$ springeropen.com 